\title{
Comparison of efficacy of systemic antibiotics alone and combination of systemic antibiotics with gentamicin cream in diabetic foot infections
}

\author{
Munawer Latif Memon', Muhammad Ikram², Muhammad Azhar ${ }^{3}$, Varda Balouch ${ }^{4}$
}

\begin{abstract}
Objective: To compare efficacy of systemic antibiotics alone and combination of systemic antibiotics with gentamicin cream in diabetic foot infections.

Methods: Prospective Observational Study was conducted at Department of Surgery, Pakistan Ordinance Factories (POF) Hospital, Wah Cantt for duration of two years (January 2018-December 2019). A sample size of 140 diabetic foot infection patients (70 patients in each group) was calculated using WHO calculator. DFI patients were selected through non probability (consecutive) sampling technique. All patients signed consent forms before participation into study. Patients were randomly divided into two group (computer generated random number table); Group-A patients were given systemic antibiotics alone while Group-B was given combination of gentamicin cream and systemic antibiotics. SPSS version 24 was utilized for analysis purpose. Chi-square test was applied in our study. Results with $p$-value $\leq 0.05$ found significant.

Results: Total 140 patients were included in study. There were $87(62.1 \%)$ male and $53(37.9 \%)$ females in our data. Mean age of patients was 46 years \pm 11 .3SD. Group-B (combination of gentamicin and systemic antibiotics) showed significant reduction in inflammation $(p=0.03)$, culture negativity $(p=0.001)$, increase clinical cure $(p=0.02)$ and pathological eradication $(p=0.03)$ as compared to Group-A (systemic antibiotics alone). Gender, age and duration of diabetes mellitus had insignificant association with outcomes ( $p>0.05)$. Conclusion: Diabetic foot infections are significant contributors of morbidity in our country. Combination of gentamicin cream with systemic antibiotics is highly effective in inflammation reduction, increasing clinical cure rate and pathological eradication as compared to systemic antibiotics alone in diabetic foot infections. Early identification of risk factors, proper patients care and multidisciplinary approach for diabetic foot infections prevention is required.
\end{abstract}

KEYWORDS: Diabetic foot infection, Gentamicin, Systemic antibiotics.

doi: https://doi.org/10.12669/pjms.38.3.3277

How to cite this:

Memon ML, Ikram M, Azhar M, Balouch V. Comparison of efficacy of systemic antibiotics alone and combination of systemic antibiotics with gentamicin cream in diabetic foot infections. Pak J Med Sci. 2022;38(3):663-667.

doi: https://doi.org/10.12669/pjms.38.3.3277

This is an Open Access article distributed under the terms of the Creative Commons Attribution License (http://creativecommons.org/licenses/by/3.0), which permits unrestricted use, distribution, and reproduction in any medium, provided the original work is properly cited.

1. Muawer Latif Memon,

Assistant Professor Surgery,

2. Muhammad Ikram,

Assistant Professor Orthopedics,

3. Muhammad Azhar,

Assistant Professor Surgery,

4. Varda Balouch

Assistant Professor Anaesthesia,

1-4: POF Hospital, Wah Cantt, Pakistan.

Correspondence:

Muawer Latif Memon,

Assistant Professor Surgery, POF Hospital Wah Cantt, Pakistan.

Postal Address: House No. 254,

MR-6, B Block, Multigardens B-17, Islamabad, Pakistan.

Email: sulehrionline99@hotmail.com

* Received for Publication:

July 14, 2020

* $1^{\text {st }}$ Revision Received:

* $2^{\text {nd }}$ Revision Received:

* Final Revision Accepted:
December 16, 2020

September 30, 2021

October 20, 2021

\section{INTRODUCTION}

Diabetic foot infections (DFI) are most common infections associated with high morbidity, worldwide. ${ }^{1}$ An estimated 25 million individuals are suffering with diabetes mellitus, out of which $15 \%$ to $25 \%$ are infected with foot ulcerations. ${ }^{2}$ Literature reported that $50 \%$ of these ulcerations lead to increased morbidity, high rate of hospitalization and lower extremity amputation due to infections. ${ }^{3}$ Diabetic foot infections account for $20 \%$ of total hospital admissions in United States. ${ }^{4}$ In Pakistan, DFI leads to $21-48 \%$ of foot amputation due to improper management and poor glycemic controls. ${ }^{5}$ 
Pathophysiology of DFI is associated with several factors including vasculopathy, neuropathy and immunopathy. Risk factors for DFI include wounds with greater than 30 days duration, wounds with traumatic etiology, bone penetrating wounds, recurrent wounds, elevated body mass index, socioeconomic factors, duration of diabetes mellitus and presence of peripheral arterial diseases (PAD). ${ }^{6}$ DFI diagnosis is usually based on clinical findings of patient. Infectious disease society of America (IDSA) reported that two or more signs of inflammation (tenderness, warmth, erythema, induration and pain) and if there is no obvious purulent drainage. Diagnosis could be based upon local and systemic infections. Local signs include pain, purulent drainage, erythema, tenderness, edema and malodor; however, systemic infections include vomiting, nausea, chills, anorexia, worse glycemic control and change in mental status. ${ }^{7}$

There are several classifications for diabetic foot infections, however, Wagner's classification is universally accepted grading system $(0=$ pre ulcerative area without open lesion, $1=$ superficial ulcer, 2=ulcer deep to tendon, capsule, bone, $3=$ stage 2 with abscess, osteomyelitis or joint sepsis, $4=$ localized gangrene and $5=$ global foot gangrene). According to IDSA, treatment/ management of DFI is based upon severity of infection and pathological agents. ${ }^{8}$

Uckay et al. reported that gentamicin sponge is effective in total clinical cure and show significant improvement in complete eradication of pathogens as compared to control $(\mathrm{p}<0.05) .{ }^{9}$ Uckey et al. conducted another study on gentamicin efficacy and reported that this topical antibiotic therapy achieve $91 \%$ clinical cure and 9\% improvement in microbiological outcomes, however, it does not improve outcomes among patients with mild DFI. ${ }^{9}$ Gentamicin is an important drug in DFI treatment; however, limited data is available on its efficacy in Pakistan. Present study aims to compare efficacy of systemic antibiotics alone and combination of systemic antibiotics with gentamicin cream in diabetic foot infections.

\section{METHODS}

This prospective observational study was conducted at Department of Surgery, Pakistan Ordinance Factories (POF) Hospital, Wah Cantt after ethical approval (Ref No.: POFHosp/Eth.com/201; dated November 13, 2020). Study duration was two (January 2018-December 2019). Sample size of 126 was rounded off to 140 DFI patients (70 patients in each group) was calculated with 95\% confidence interval, power of study $84 \%, \mathrm{P} 1=20 \%$ and $\mathrm{P} 2=15 \%$ using WHO calculator. ${ }^{10}$ Sampling was done with non-probability (consecutive) sampling technique. Inclusion criteria was based upon age 18-70 years, both genders and diagnosed with diabetic foot infection (based on IDSA criteria), patients with $\geq 1 \mathrm{~cm}^{2}$ wound (below the malleolus), non-lactating, non-pregnant women and patients undergone any surgical intervention required for infected or necrotic tissue removal. Patients with osteomyelitis, proven ischemia on clinical examination and duplex scanning, patients with severe immune suppressions, extensive necrosis requiring amputation, peripheral arterial insufficiency requiring revascularization, infection due to any implant or foreign material insertion, patients already using gentamicin, alcohol or substance abusers were excluded from study. Diabetic foot infections were defined as presence of at least two signs of inflammation (erythema, warmth, swelling, tenderness), purulent discharge from ulcer or nearby sinus tract, along with culture positive wound swab. Clinical cure was defined as absence of at least two inflammation signs, purulent discharge and negative culture swab after intervention. ${ }^{4}$ Patients were randomly divided into two groups; Group-A was given systemic quinolone (ciprofloxacin 200mg 12hrly) while Group-B was given topical gentamicin cream (amount of $10 \mathrm{~g}$ of $0.1 \% \mathrm{w} / \mathrm{v}$ gentamicin /gentamicin sulphate depending upon wound size) along systemic antibiotic in the form of quinolone (ciprofloxacin 200mg 12hrly). Patients were followed for seven days. Efficacy of treatment was measured in terms of inflammation reduction (50\% from baseline), culture results (negative), clinical cure of infection (50\% from baseline) and microbiological eradication (50\% from baseline measurement) using clinical and laboratory standard institute (CLSI) guidelines. SPSS version 24 was used for data analysis. Mean and standard deviation was calculated for numerical (quantitative) data while categorical and nominal data was analyzed in terms of frequencies and percentages. Effect modifiers like age and gender were controlled using stratification process. Post stratification chi-square was utilized for measuring association between different variables. P-value $\leq 0.05$ was reported as statistically significant difference in both interventions.

\section{RESULTS}

Total 140 patients were included in study. There were $87(62.1 \%)$ male and $53(37.9 \%)$ females in our 


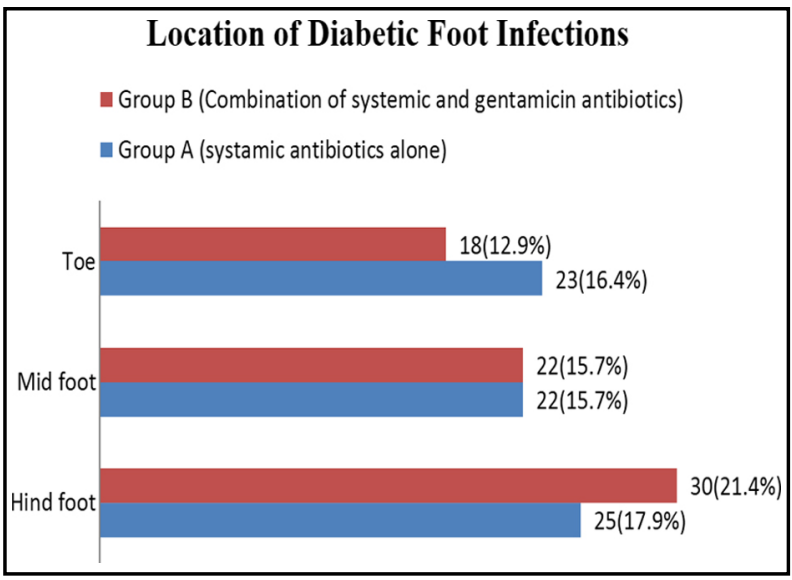

Fig.1: Location of diabetic foot infection.

data. Mean age of patients was 46 years $\pm 11.3 S D$. There were $46(32.9 \%)$ patients in age group 18 40 years and $94(67.1 \%)$ patients in age group $41-$ 70 years. Duration of diabetes mellitus was $\leq 6$ months in $56(40 \%)$ and $84(60 \%)$ had $>6$ months of diabetes duration.

Location of diabetic foot infection in Group-A was hind foot $17.9 \%$, mid foot in $15.7 \%$, and toe in $16.4 \%$ while in Group-B DFI was located in hind foot $21.4 \%$, mid foot in $15.7 \%$ and toe in $12.9 \%$ patients as shown in Fig.1.

Organism causing DFI were staphylococcus aureus $(10.7 \%$ vs $13.6 \%)$, pseudomonas aeruginosa (10\% vs $6.4 \%)$, S. epidermidis ( $12.1 \%$ vs $10 \%)$ and polymicrobes (17.1\% vs $20 \%)$ in Group-A and Group-B respectively as shown in Fig.2. Among all the patients in Group-A 70(50\%), inflammation reduction was seen in 17(12.1\%) and inflammation was not reduced in 53(37.9\%). Among all the patients in Group-B 70(50\%), inflammation was reduced in 29(20.7\%) patients and not reduced in $41(29.3 \%)(p=0.03)$. In Group-A, Clinical cure was seen in $13(9.3 \%)$ and not observed in $57(40.7 \%)$

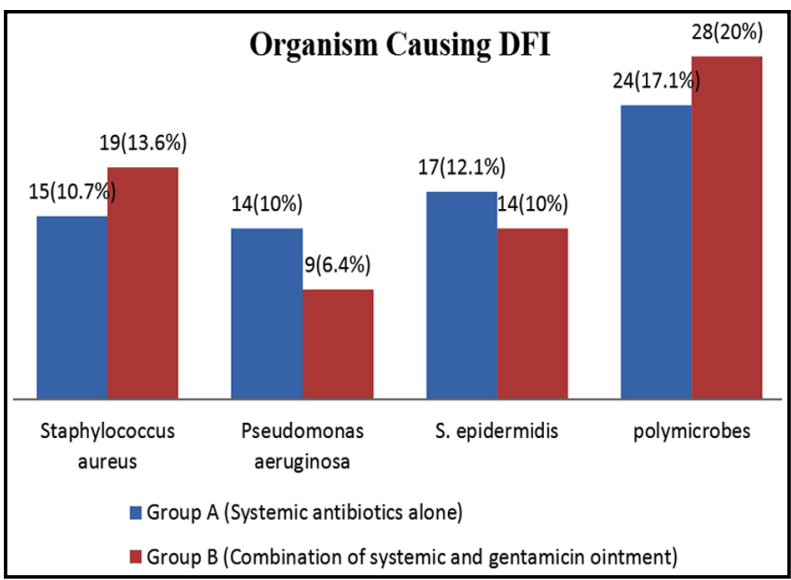

Fig.2: Organism causing DFI.

patients. In Group-B, 26(18.6\%) patients show clinical cure while $44(31.4 \%)$ did not show clinical cure $(\mathrm{p}=0.02)$ as shown in Table-I.

After intervention culture results were negative in $22(15.7 \%)$ and positive in $48(34.3 \%)$ patients in Group-A while results were negative in $42(30 \%)$ patients and positive in $28(20 \%)$ patients in Group-B $(\mathrm{p}=0.001)$. Pathological eradication was found in $21(15 \%)$ and not eradicated in $49(35 \%)$ patients in Group-A while in Group-B pathological eradication was reported in $34(24.3 \%)$ patients and not eradicated in $36(25.7 \%)$ patients $(\mathrm{p}=0.03)$ as shown in Table-II.

\section{DISCUSSION}

Diabetic foot infections are most common complication of diabetes foot ulceration. Pakistan is among top 10 countries affected with diabetes mellitus leading to high incidence of diabetic foot infection. ${ }^{11}$ Jan et al. reported that foot is advise and management is an important challenge for not only diabetic patients but also for health care professionals managing diabetic foot infections. ${ }^{12}$

Table-I: Comparison of inflammation reduction and clinical cure in both groups.

\begin{tabular}{lcccc}
\hline \multirow{2}{*}{ Efficacy } & \multicolumn{2}{c}{ Interventional Groups } & Total & P-value \\
\cline { 2 - 4 } Inflammation reduction & $\begin{array}{c}\text { Group-A } \\
\text { (Systemic antibiotics alone) }\end{array}$ & $\begin{array}{c}\text { Group-B (Combination of systemic } \\
\text { and gentamicin antibiotic) }\end{array}$ & & \\
\hline No & $53(37.9 \%)$ & $41(29.3 \%)$ & $94(67.1 \%)$ & 0.03 \\
Yes & $17(12.1 \%)$ & $29(20.7 \%)$ & $46(32.9 \%)$ & \\
Clinical cure & & & & \\
No & $57(40.7 \%)$ & $44(31.4 \%)$ & $101(72.1 \%)$ & 0.02 \\
Yes & $13(9.3 \%)$ & $26(18.6 \%)$ & $39(27.9 \%)$ & \\
\hline Total & $70(50 \%)$ & $70(50 \%)$ & $140(100 \%)$ & \\
\hline
\end{tabular}


Table-II: Comparison of culture results and pathological eradication in both groups.

\begin{tabular}{|c|c|c|c|c|}
\hline \multirow[b]{2}{*}{ Efficacy } & \multicolumn{2}{|c|}{ Interventional Groups } & \multirow[t]{2}{*}{ Total } & \multirow[t]{2}{*}{ Pvalue } \\
\hline & $\begin{array}{c}\text { Group }-A \\
\text { (Systemic antibiotics alone) }\end{array}$ & $\begin{array}{c}\text { Group-B (Combination of systemic } \\
\text { and gentamicin antibiotic) }\end{array}$ & & \\
\hline \multicolumn{5}{|c|}{ Culture results } \\
\hline Negative & $22(15.7 \%)$ & $42(30 \%)$ & $64(45.7 \%)$ & 0.001 \\
\hline Positive & $48(34.3 \%)$ & $28(20 \%)$ & $76(54.3 \%)$ & \\
\hline \multicolumn{5}{|c|}{ Pathological eradication } \\
\hline No & $49(35 \%)$ & $36(25.7 \%)$ & $85(60.7 \%)$ & 0.03 \\
\hline Yes & $21(15 \%)$ & $34(24.3 \%)$ & $55(39.3 \%)$ & \\
\hline Total & $70(50 \%)$ & $70(50 \%)$ & $140(100 \%)$ & \\
\hline
\end{tabular}

In present study, Group-B (combination of gentamicin and systemic antibiotics) showed significant reduction in inflammation as compared to Group-A (systemic antibiotics alone) (20.7\% vs $12.1 \%, \mathrm{p}=0.03$ ). Chu et al reported that topical antibiotics are effective in DFI depending upon site of infection and prevent systemic side effects. ${ }^{13}$ Landsman et al. reported that gentamicin topical ointments are effective in small, mild and superficial diabetic foot infection, however, their efficacy is limited in severe infections. ${ }^{14}$

In present study, Group-B had high clinical cure rate as compared to Group-A (18.6\% vs $9.3 \%$, $\mathrm{p}=0.02)$. Lipsky et al. reported that gentamicin sponge is showed $35 \%$ of clinical cure. Moreover, gentamicin sponge are safe, well tolerated and without any attributed side effects. ${ }^{15}$ Creanor et al. reported that gentamicin had safe role in treatment of diabetic foot infection while gentamicin did not show effective results as adjunctive therapy resulting in demand of larger clinical trials. ${ }^{16}$

Statistically significant reduction in pathological eradication was seen in Group-B as compared to Group-A (24.3\% vs $15 \%, \mathrm{p}=0.03)$. Dumville et al. reported that total eradication of pathogens was seen in $52 \%$ patients using gentamicin collagen sponge. ${ }^{17}$ Varga et al. reported that gentamicin is more effective in antimicrobial eradication in topical form due to direct interaction with infected site as compared to systemic route. ${ }^{18}$ Another similar study reported that gentamicin ointments in combination with systemic antibiotics leads to better diabetic foot infections outcomes, however, long term efficacy of treatment is limited. ${ }^{19}$

In present study, Organism causing DFI were staphylococcus aureus $(10.7 \%$ vs. $13.6 \%)$, pseudomonas aeruginosa $(10 \%$ vs $6.4 \%)$, S. epidermidis $(12.1 \%$ vs $10 \%)$ and polymicrobes
$(17.1 \%$ vs $20 \%)$ in Group-A and Group-B respectively. Charles et al. reported that poly microbial infections are most common in DFI (83\%) including patients with Wagner grade 3 and $4 .{ }^{20}$ Reber et al. reported that gram negative organism E.Coli and S.aureus are most frequent pathogens associated with DFI (63\% and $58 \%$ respectively). ${ }^{21}$ Miyan et al. reported that diabetic foot infections are associated with gram negative aerobes. They lead to conclusion that delayed referral is major cause of increasing frequency of multiple drug resistance isolates. ${ }^{22}$ Alavi et al. reported that in their data common causes of DFI were Escherichia Coli, Proteus vulgaris and Staphylococcus aureus with an antibiotic resistance $65 \% .^{23}$

Diabetic foot infections are leading cause of morbidity in Pakistan. There is a lot of literature available on this topic internationally. However, to the best of our knowledge this study is a unique study in Pakistan. We recommend use of systemic antibiotics in combination with gentamicin cream for diabetic foot infection treatment in resource limited areas.

Limitation of the study: Conduction of study at single center limits generalization of study.

\section{CONCLUSION}

Diabetic foot infections are significant contributors of morbidity in our country. Combination of gentamicin cream with systemic antibiotics is highly effective in inflammation reduction, increasing clinical cure rate and pathological eradication as compared to systemic antibiotics alone in diabetic foot infections. Early identification of risk factors, proper patients care and multidisciplinary approach for diabetic foot infections prevention is required. 
Study contribution to medical field: Diabetes is very common now in Pakistan. Diabetic foot infections lead to serious morbidity. It's very important to understand efficacy of treatment at local level in Pakistan for diabetic foot infection. It will help the physicians to choose a treatment that is easy and feasible for diabetic patients.

Conflict of interest: None.

Source of funding: None.

\section{REFERENCES}

1. Bakker K, Schaper NC. The development of global consensus guidelines on the management and prevention of the diabetic foot 2011. Diabetes Metab Res Rev. 2012;28(1):116-118.

2. Boulton AJM.The pathway to foot ulceration in diabetes. Med Clin North Am. 2013;97(5):775-790. doi: 10.1016/j. mona.2013.03.007

3. Lavery LA, Hunt NA, LaFontaine J, Baxter CL, Ndip A, Boulton AJ. Diabetic foot prevention: a neglected opportunity in high-risk patients. Diabetes Care. 2010;33(7):1460-1462.

4. Tiwari S, Pratyush DD, Dwivedi A, Gupta SK, Rai M, Singh SK. Microbiological and clinical characteristics of diabetic foot infections in northern India. J Infect Dev Ctries. 2012;6(4):329-332. doi: 10.3855/jidc.1827

5. Kaimkhani GM, Siddiqui AA, Rasheed N, Rajput MI, Kumar J, Khan MH et al. Pattern of Infecting Microorganisms and Their Susceptibility to Antimicrobial Drugs in Patients with Diabetic Foot Infections in a Tertiary Care Hospital in Karachi, Pakistan. Cureus. 2018;10(6):2872. doi: 10.7759/ cureus.2872

6. Peter-Riesch B. The diabetic foot: the never-ending challenge. Endocr Dev. 2016;31(2):108-134. doi 10.1159/000439409

7. Lipsky BA, Aragón-Sánchez J, Diggle M. International Working Group on the Diabetic Foot. IWGDF guidance on the diagnosis and management of foot infections in persons with diabetes. Diabetes Metab Res Rev.2016;32(3):45-74. doi: 10.1002/dmrr.2699

8. Neidrauer M, Zubkov L, Weingarten MS, Pourrezaei K, Papazoglou ES. Near infrared wound monitor helps clinical assessment of diabetic foot ulcers. J Diabetes Sci Technol. 2010;4(3):792-798. doi: 10.1177/193229681000400404

9. Uckay I, Kressmann B, Malacarne S, Toumanova A, Jaafar J, Lew D et al. A randomized, controlled study to investigate the efficacy and safety of a topical gentamicincollagen sponge in combination with systemic antibiotic therapy in diabetic patients with a moderate or severe foot ulcer infection. BMC Infect Dis. 2018;18(1):361. doi: 10.1186/s12879-018-3253-z

10. Markowitz JS, Gutterman EM, Magee G, Margolis DJ. Risk of amputation in patients with diabetic foot ulcers: a claims-based study. Wound Repair Regen. 2006;14(2):1117. doi: $10.1111 /$ j.1743-6109.2005.00083.x

11. Jawaid AS, Jawaid M. Management of diabetic foot ulcers: some bitter facts and harsh realities. Pak J Med Sci. 2006;22(2):97-100.

12. Jan AW, Khan H, Ahmad I, Khan M. Diabetic foot ulcer; risk factors stratification in patients. A study of 150 patients. Prof Med J. 2016;23(3):693-698.
13. Chu Y, Wang C, Zhang J. Can we stop antibiotic therapy when signs and symptoms have resolved in diabetic foot infection patients? Int J Low Extrem Wounds. 2015;14(4):277-283. doi: 10.1177/1534734615596891

14. Landsman A, Blume PA, Jordan DA, Jr. An open-label, three-arm pilot study of the safety and efficacy of topical Microcyn Rx wound care versus oral levofloxacin versus combined therapy for mild diabetic foot infections. J Am Podiatr Med Assoc. 2015;101(7):484-496. doi: 10.7547/1010484

15. Lipsky BA, Kuss M, Edmonds M. Topical application of a gentamicin-collagen sponge combined with systemic antibiotic therapy for the treatment of diabetic foot infections of moderate severity: a randomized, controlled, multicenter clinical trial. J Am Podiatr Med Assoc. 2012;102(3):223-232. doi: 10.7547/1020223

16. Creanor S, Barton A, Marchbank A. Effectiveness of a gentamicin impregnated collagen sponge on reducing sterna wound infections following cardiac surgery: a meta-analysis of randomized controlled trials. Ann R Coll Surg Engl. 2012;94(3):227-231. doi: 10.1308/003588412X1 3171221590179

17. Dumville JC, Lipsky BA, Hoey C. Topical antimicrobial agents for treating foot ulcers in people with diabetes. Cochrane Database Syst Rev. 2017;6(2):CD011038. doi: 10.1002/14651858.CD011038.pub2

18. Varga M, Sixta B, Bem R, Matia I, Jirkovska A, Adamec M. Application of gentamicin-collagen sponge shortened wound healing time after minor amputations in diabetic patients - a prospective, randomised trial. Arch Med Sci. 2014;10(2):283-287. doi: 10.5114/aoms.2014.42580.

19. Uxkay I, Aragon-Sanchez J, Lew D, Lipsky BA. Diabetic foot infections: what have we learned in the last 30 years? Int J Infect Dis. 2015;40(2):81-91. doi: 10.1016/j. ijid.2015.09.023

20. Charles PG, Uçkay I, Kressmann B, Emonet S, Lipsky BA. The role of anaerobes in diabetic foot infections. Anaerobe. 2015;34(4):8-13. doi: 10.1016/j.anaerobe.2015.03.009.

21. Reber A, Moldovan A, Dunkel N, Emonet S, Rohner $\mathrm{P}$, Tahintzi $\mathrm{P}$, et al. Should the methicillin-resistant Staphylococcus aureus carriage status be used as a guide to treatment for skin and soft tissue infections? J Inf Secur. 2012;64(3):513-519. doi: 10.1016/j.jinf.2011.12.023

22. Miyan Z, Fawwad A, Sabir R, Basit A. Microbiological pattern of diabetic foot infections at a tertiary care center in a developing country. J Pak Med Assoc. 2017;67(5):665-669.

23. Alavi MS, Khosravi DA, Sarami A, Dashtebozorg A, Montazeri AE. Bacteriologic study of diabetic foot ulcer. Pak J Med Sci. 2007;23(5):681-684.

\section{Authors' Contribution:}

MLM: Data collection, study designing, responsible for accuracy and integrity of study. MI: Data analysis and interpretation of results. MA: Data acquisition, Critical evaluation of intellectual content.

VB: Study write ups, Interpretation of data and critical review. 